\title{
Isolated liver trauma: A clinical perspective in a non-emergency center for liver surgery
}

\author{
STEFAN ANDREI $^{1,2}$, SEBASTIAN ISAC $^{1,3}$, MARICICA CARSTEA $^{1}$, CRISTINA MARTAC $^{1}$, \\ LUCIAN MIHALCEA $^{1}$, CRISTINA BUZATU ${ }^{1}$, DORIN IONESCU ${ }^{4,5}$, \\ DRAGOS EUGEN GEORGESCU ${ }^{6}$ and GABRIELA DROC ${ }^{1,2}$
}

\author{
${ }^{1}$ Department of Anesthesiology and Intensive Care I, 'Fundeni' Clinical Institute, 022328 Bucharest; \\ Departments of ${ }^{2}$ Anesthesiology and Intensive Care, Faculty of Medicine, ${ }^{3}$ Physiology II and Neurosciences, \\ Faculty of Medicine, and ${ }^{4}$ Medical Semiology, Discipline of Internal Medicine I and Nephrology, Faculty of Medicine, \\ 'Carol Davila' University of Medicine and Pharmacy, 020021 Bucharest; ${ }^{5}$ Department of Nephrology, \\ Bucharest Emergency University Hospital, 050098 Bucharest; ${ }^{6}$ Department of Surgery, Faculty of Medicine, \\ 'Carol Davila' University of Medicine and Pharmacy, 020021 Bucharest, Romania
}

Received August 20,2021; Accepted September 21, 2021

DOI: $10.3892 / \mathrm{etm} .2021 .10961$

\begin{abstract}
The management of liver trauma is, currently, still heterogeneous ranging from conservative to major invasive liver resections. When appropriate, these cases should be referred to a regional care center. The objective of this study was to analyze the expertise of a non-emergency center for liver surgery from Romania after initial stabilization in county hospitals. This study is a monocentric, retrospective, observational study, including 12 patients with hepatic trauma after a car accident, admitted between 2015 and 2019. We analyzed various clinical and biochemical data as independent variables, and the main outcome was considered the intensive care unit (ICU) length of stay. Our results revealed that intubation status at admission, norepinephrine infusion during surgery, hyperfibrinogenemia and duration of mechanical ventilation in patients with isolated liver trauma were correlated with prolonged ICU length of stay. Further prospective, more
\end{abstract}

Correspondence to: Dr Sebastian Isac, Department of Anesthesiology and Intensive Care I, 'Fundeni' Clinical Institute, 258 Soseaua Fundeni, 022328 Bucharest, Romania

E-mail: sebastian.isac@umfcd.ro

Abbreviations: APACHE II, Acute Physiology and Chronic Health Evaluation II score; ASA, American Society of Anesthesiology score; BMI, Body mass index; BUN, blood urea nitrogen; CVVHDF, continuous veno-venous hemodiafiltration; FFP, fresh frozen plasma; ICU, intensive care unit; ISS, injury severity score; NE, norepinephrine; PC, platelet concentrate; SOFA, Sepsis-related Organ Failure Assessment; TB, total bilirubin; WHO, World Health Organization

Key words: liver trauma, ICU stay, hyperfibrinogenemia, mechanical ventilation, norepinephrine comprehensive studies are needed in order to evaluate the exact prognostic factors in terms of short- and long-term mortality.

\section{Introduction}

According to the World Health Organization (WHO), 5.8 million people die each year as a result of injuries, being responsible for $10 \%$ of the world's mortality (1). Despite the burden, there are disparities, globally, regarding trauma care (2).

Liver trauma is not an uncommon lesion with life-threatening potential in trauma patients, which can be managed with either operative or non-operative strategy, according to the severity, patient characteristics and trauma mechanism $(3,4)$.

An increased non-surgical approach has been noted (5). However, the management is challenged by the lack of definitive studies and by the worldwide heterogeneity (6). Therapy management is difficult, being complicated by the risk of hemorrhagic shock, liver failure, sepsis and organ dysfunction. The decision is to be made by a multidisciplinary team able to deal with a complex patient: intensive care unit (ICU) specialists, surgeons of different specialties, interventional radiologist in specialized tertiary center. Usually, these complex patients are managed according to local logistics and personnel resources, being addressed either in specialized trauma centers, but also in county hospitals in accordance with the clinical status or transport availability. When considered appropriate, these patients are transferred to a hepatic center (7).

The objective of this study was to analyze the outcomes of the patients with post-traumatic liver injury requiring secondary ICU hospitalization in a non-emergency liver center from Romania after initial stabilization in county hospitals.

\section{Patients and methods}

Study design and data collection. This study is a monocentric, retrospective, observational study, designed in a clinical 
center for hepatobiliary surgery. Patients with hepatic trauma after car accident, admitted between 2015 and 2019, were included. The data were collected using the electronic medical record as the patients' paper charts. There were 5 females and 7 males with a mean age of 27 years and a mean body mass index (BMI) of $23.1 \mathrm{~kg} / \mathrm{m}^{2}$.

The following variables were noted: age, sex, intubation status at admission, Acute Physiology and Chronic Health Evaluation (APACHE) II score at admission, BMI, trauma-admission time, admission-surgery time, surgical procedure before transfer (in the primary hospital), injury severity score (ISS), Sepsis-related Organ Failure Assessment (SOFA) at 24, 48 and $72 \mathrm{~h}$ postadmission respectively, norepinephrine (NE) infusion rate at admission, and postoperatively, the need for continuous veno-venous hemodiafiltration (CVVHDF), preoperative standard blood analysis, thromboelastographic analysis before, during and after surgery, perioperative lactate dynamics, need of preoperative transfusion, if reintervention was needed, type of surgery, duration of surgery, blood products needed during and after surgery, hours of mechanical ventilation cumulated during the ICU admission, and the length of ICU stay.

The standard blood analysis consisted of: full blood cell count, liver enzymes, urea nitrogen (BUN), creatinine, total bilirubin (TB), fibrinogen, thromboelastographic parameters. We considered the following surgery types: demeshing (after successful meshing in primary hospitals, where they were initially admitted), partial liver resection, or partial liver resection with supplementary surgical procedures to other abdominal or thoracic organs.

We considered blood products and antifibrinolytics administered during ICU stay and included separately all of the following: erythrocyte concentrate, fresh frozen plasma (FFP), platelet concentrate (PC), fibrinogen, and tranexamic acid.

Ethic statement. All clinical procedures were carried out with the approval of the local Ethics Committee of 'Fundeni' Clinical Institute (Romania) for clinical trials in accordance with the European Communities Council Directive 2001/20/EC and with respect to personal data privacy, European Directive 95/46/EC. Written informed consent was obtained from all patients prior to publication.

Data analysis. Data analysis and graphical representations were performed using GraphPad Prism 6.00 (GraphPad Software Inc.).

For the correlative data, we used a linear correlation model, between the number of hospitalization days as outcome variable and all other variables as independent variables. The Spearman correlation coefficient, $r$, was determined. We stated a $r$ value between -0.3 and 0.3 to have negligible correlative effect.

Furthermore, for the qualitative independent variables, which influenced statistically significantly the number of hospitalization days, we compared the median of the hospitalization days between groups (with or without the specified independent variable) using Mann-Whitney test analysis after assessing the groups for normal distribution using histograms, D' Agostino-Pearson omnibus normality test and Shapiro-Wilk test. A two-sided P-value $<0.05$ was considered statistically significant.

\section{Results}

A total of 12 patients were enrolled and 11 were included in the analysis, considering that for 1 patient the data collection was incomplete.

Correlations between the length of ICU stay and the independent variables considered. The length of ICU hospitalization was found to depend mainly on intubation status at admission [rho (r) $=0.6992, \mathrm{P}=0.0303$ ], the cumulative number of mechanical ventilation hours $(\mathrm{r}=0.6713, \mathrm{P}=0.0273)$, preoperative fibrinogen values $(\mathrm{r}=0.7301, \mathrm{P}=0.0208)$ and the $\mathrm{NE}$ intraoperative use $(\mathrm{r}=0.8157, \mathrm{P}=0.0087)$. The Spearman correlation coefficient, rho, and P-value for each correlation between the length of ICU hospitalization and those independent variables considered to have a potential correlative effect (Spearman correlation coefficient outside the interval -0.3-0.3) are documented in Table I.

Comparative analysis of the length of ICU stay considering the intubation status at admission and the use of NE intraoperatively. The median hospitalization days was increased in admitted intubated patients and in patients who received NE during surgery $(\mathrm{r}=0.81, \mathrm{P}=0.0087)$ (Table $\mathrm{I}$ ). The data are represented in Fig. 1. The graphs represent the ranks, median and $95 \% \mathrm{CI}$ of the groups. The median ICU stay in patients with no NE during surgery was 3 days (95\% CI: 2.14-6.65) compared with those patients, who needed NE during surgery: 8 days (95\% CI: 4.9-23.1) (Fig. 1A). The median ICU stay in non-intubated patients at admission was 5 days (95\% CI: 2.18-7.02), while for those intubated at admission was 8 days (95\% CI: 4.53-23.14) (Fig. 1B).

\section{Discussion}

The analysis included all the patients treated after initial stabilization in a highly specialized center from Eastern Europe. The small number of patients precluded any inference about mortality. Thus, we used as the main outcome, the length of ICU stay. According to the literature, there are various major risk factors associated with mortality in this clinical setting: multiple trauma, extra-abdominal lesions, initial systolic blood pressure, initial base deficit, the Glasgow Coma Scale, injury type, number of resected segments, and intraoperative blood loss (8).

We observed that patients, who needed intraoperative vasopressors, had a longer ICU stay, despite the fact that no patient needed norepinephrine after the surgical procedure, probably a marker of severity.

The use of vasopressors is a straight-forward decision in patients with profound hypotension in order to maintain an adequate perfusion pressure in vital organs $(9,10)$. Their use should be, however, timely-limited until the main cause is identified and treated. In accordance with advanced trauma life support management, the use of vasopressors (especially norepinephrine) in the early stage of trauma could be deleterious (11). Fluid resuscitation should be prioritized in order to maintain the main blood pressure in hemorrhagic shock. The extensive use of vasopressors intraoperatively impairs 
Table I. Correlations between the length of ICU stay and the independent variables considered.

\begin{tabular}{lrr}
\hline Characteristics & Rho (r) & P-value \\
\hline Duration of mechanical ventilation (h) & 0.6713 & $\mathbf{0 . 0 2 7 3}$ \\
Trauma admission time & -0.3936 & 0.2937 \\
Admission-surgery time & -0.3929 & 0.2889 \\
ISS & 0.5576 & 0.0786 \\
SOFA (admission) & 0.3733 & 0.2574 \\
SOFA (72 h after admission) & 0.3801 & 0.2787 \\
Intubation status & 0.6992 & $\mathbf{0 . 0 3 0 3}$ \\
Hgb & 0.3262 & 0.3561 \\
Platelet count & 0.5338 & 0.1184 \\
Fibrinogen & 0.7301 & $\mathbf{0 . 0 2 0 8}$ \\
INR & -0.3497 & 0.3251 \\
CT fib & -0.3172 & 0.4554 \\
Lactate at admission & -0.4356 & 0.2123 \\
Transfusion on admission & -0.3619 & 0.3061 \\
Revision surgery & 0.3016 & 0.4121 \\
PC transfusion & 0.6292 & 0.1071 \\
NE intraoperative use & 0.8157 & $\mathbf{0 . 0 0 8 7}$ \\
CVVHDF & 0.5238 & 0.1556 \\
Postoperative transfusion & 0.5036 & 0.1762 \\
ASA & 0.3304 & 0.3245 \\
\hline
\end{tabular}

ICU, intensive care unit; ISS, injury severity score; SOFA, Sepsis-related Organ Failure Assessment; Hgb, hemoglobin; INR, international normalized ratio; PC, platelet concentrate; NE, norepinephrine; CT fib, clotting time FIBTEM (ROTEM); CVVHDF, continuous veno-venous hemodiafiltration; ASA, American Society of Anesthesiology score. Rho represents the Spearman correlation coefficient. A two-sided P-value $<0.05$ was considered statistically significant; significant $\mathrm{P}$-values are indicated in bold print.

microcirculation and oxygen balance to the vital organs, thus increasing the fatality risk (12).

Considering that our patients were fluid resuscitated prior to admission, we could advocate that the norepinephrine-dependent patients were either inadequately fluid-resuscitated and/or had a more complex liver trauma, who needed even more blood products and crystalloids, although these two factors were not associated, in our study, with a prolonged ICU stay. Microcirculation impairments or the degree of systemic inflammation due to norepinephrine use could be consequences of prolonged ICU stay. Our data are in accordance with Uchida $e t a l$, who showed that the use of norepinephrine could increase the mortality rate/ICU stay (13).

Prehospital airway protection by means of endotracheal intubation in trauma patients is a debated issue in the literature (14). The actual guidelines promote the early endotracheal intubation in polytrauma patients, especially those with thorax, abdominal and/or head injury. The decision to intubate at the scene or in the ER department should be guided by the type of trauma (15). Avoiding hypoxia along with maintaining airway patency in this patient subgroup is
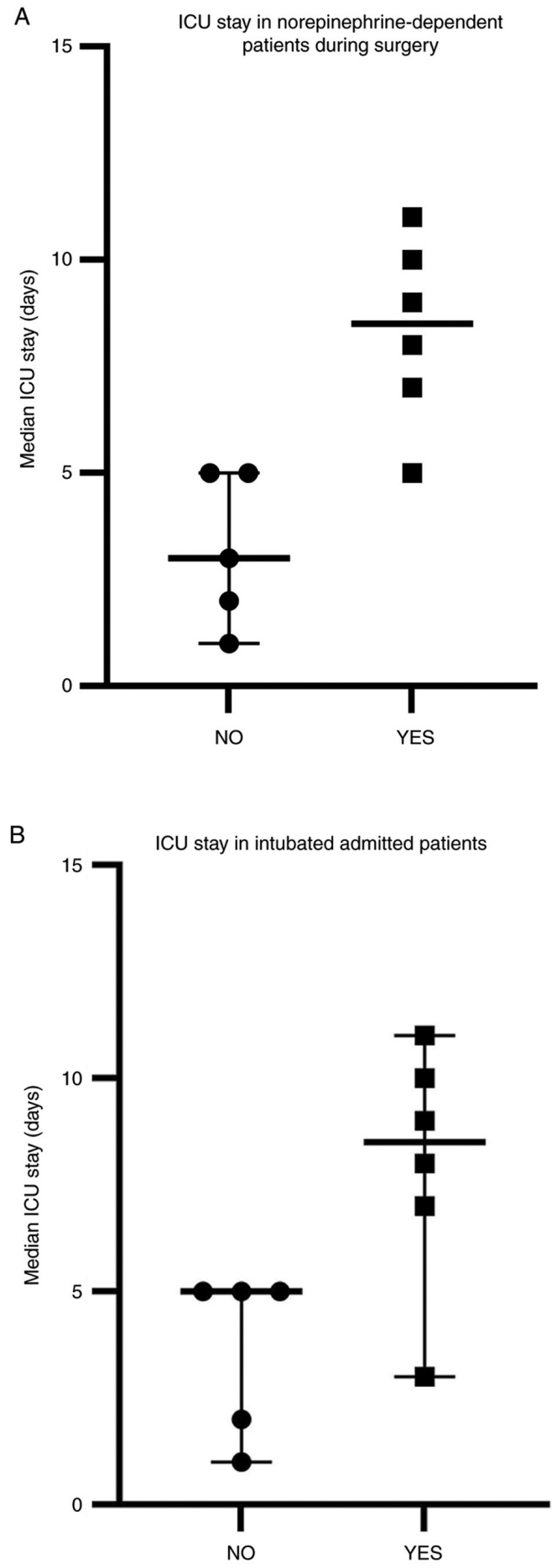

Figure 1. (A) The median ICU stay in patients with and without NE during surgery. The length of stay is expressed in days and the bars represents the median of the ICU stay. (B) The median ICU stay in non-intubated vs. intubated patients at admission. The length of stay is expressed in days and the bars represents the median of the ICU stay. ICU, intensive care unit; NE, norepinephrine.

crucial. The long-term beneficial effect, is, however, controversial (16).

Little is known about early intubation in patients with isolated traumatic liver injury. In our study, the patients with isolated liver trauma, who were intubated in prehospital settings, had a longer stay in the ICU. This finding could be explained due to various reasons: residual sedation with 
consequent postoperative prolonged weaning, increased personnel infrastructure and time-consuming perioperative diagnostic and therapeutic maneuvers related to the complexity of the case, difficulties in prehospital ICU bed allocation process, and overall case severity. Our results are in contradiction with Gravesteijn et al (16). Their study suggested that patients with abdominal trauma had a better functional outcome if intubated in prehospital settings. Further observational prospective studies are needed to evaluate this clinical approach. Additionally, we observed that ventilation hours were correlated with the ICU length of stay. This finding could be a consequence of a prolonged weaning time, acquired muscle weakness and intubation-related nursing impairments.

Mechanical ventilation time in trauma patients has been also shown to have other detrimental effects such as: longer ICU stay and hospital stay, higher rates of tracheostomy and mortality (17).

Fibrinogen may be reduced following traumatic injury due to loss from hemorrhage, increased consumption and reduced synthesis (18). Plasma fibrinogen levels have been found to decrease earlier than other hemostasis parameters in trauma patients (e.g., platelet count, prothrombin time, activated partial thromboplastin time). There are three proposed mechanisms for plasma fibrinogen decrease: coagulation activation-induced consumption, hyper-fibrino(geno) lysis-induced degradation, and dilution by infusion/transfusion. Initial fibrinogen levels less than the normal range are independently associated with higher in-hospital mortality in trauma patients (19).

In liver trauma patients, because of the destruction of the liver parenchyma, plasma fibrinogen levels can be furthermore decreased, leading to fibrinogen concentrate substitution therapy (20).

In this study, the patients with a high fibrinogen concentration at admission had a prolonged length of ICU stay. These results are in contradiction with other research (18), but there are some possible explanations. The rise of fibrinogen levels from day 2 onwards can be attributed to an upregulated fibrinogen synthesis in the liver, occurring as part of the acute phase response after tissue injury (4).

This result draws our attention to the role of fibrinogen as part of the acute phase response after tissue injury.

Study limitations. Our study has some inherent limits. First, we analyzed only a limited number of patients, who were presented to our service. This aspect had known consequences on the statistical analysis and conclusions. Secondly, the study design was retrospective and monocentric. Thus, no causality can be affirmed. In addition, some data before admission were not available for analysis and discussion for some patients. Despite these evident flaws, we have to state that our hospital is the main national center in hepatic surgery, serving the entire nation of Romania. Consequently, even in small numbers, our results have a national epidemiological interest, considering both the Romanian population, but also the Romanian healthcare organization.

This aspect is of interest, opening insights of a local Eastern European experience, but it mau also represent a further limit. The trauma transfusion guidelines can be applied in an unpredictable manner, particularly in small provincial hospitals.

In conclusion, our study revealed the experience in a national center for liver surgery, from the intensive care perspective, in isolated liver trauma, proving that intubation status at admission, intraoperative norepinephrine use, mechanical ventilation hours and plasma fibrinogen are deleterious independent variables which correlates with the ICU length of stay. Further large prospective studies are needed in order to confirm these results.

\section{Acknowledgements}

The authors would like to thank Artsiom Klimko for his help with manuscript reviewing.

\section{Funding}

No funding was received.

\section{Availability of data and materials}

Due to confidentiality reasons, data generated or analyzed during this study are not included in this published article.

\section{Authors' contributions}

SI performed the statistical analysis, wrote and reviewed the manuscript, and designed the study. SA wrote the manuscript, collected the data, and designed the study. MC collected the data, contributed to manuscript writing, designed the study; CM contributed to the manuscript writing and to study design. LM collected the data and contributed to the study design. CB reviewed the manuscript and data collection. DI contributed to the manuscript writing and to the literature and manuscript reviewing. DEG contributed to the manuscript writing and to the literature and manuscript reviewing. GD coordinated the study, contributed to the study design and reviewed the manuscript. All authors agreed on the final form of the manuscript for publication.

\section{Ethics approval and consent to participate}

This study was approved by the local Ethics Committee of 'Fundeni' Clinical Institute (Romania). Written informed consent was obtained from all patients prior to publication.

\section{Patient consent for publication}

Not applicable.

\section{Competing interests}

The authors declare that they have no competing interests.

\section{References}

1. World Health Organization (WHO) (2010) Injuries and Violence: The Facts. World Health Organization, Geneva, 2010, https://www.who.int/violence_injury_prevention/en/. 
2. Sakran JV, Greer SE, Werlin E and McCunn M: Care of the injured worldwide: Trauma still the neglected disease of modern society. Scand J Trauma Resusc Emerg Med 20: 64, 2012.

3. Park KB, You DD, Hong TH, Heo JM and Won YS: Comparison between operative versus non-operative management of traumatic liver injury. Korean J Hepatobiliary Pancreat Surg 19: 103-108, 2015.

4. Coccolini F, Coimbra R, Ordonez C, Kluger Y, Vega F, Moore EE, Biffl W, Peitzman A, Horer T, Abu-Zidan FM, et al: Liver trauma: WSES 2020 guidelines. World J Emerg Surg 15: 24, 2020.

5. David Richardson J, Franklin GA, Lukan JK, Carrillo EH, Spain DA, Miller FB, Wilson MA, Polk HC Jr and Flint LM: Evolution in the management of hepatic trauma: A 25-year perspective. Ann Surg 232: 324-330, 2000.

6. Cirocchi R, Trastulli S, Pressi E, Farinella E, Avenia S, Morales Uribe $\mathrm{CH}$, Botero $\mathrm{AM}$ and Barrera LM Non-operative management versus operative management in high-grade blunt hepatic injury. Cochrane Database Syst Rev (issue 8): CD010989, 2015.

7. Popescu I, Ionescu M, Braşoveanu V, Hrehoreţ D, Copca N, Lupaşcu C, Botea F, Dorobanţu B, Alexandrescu S, Grigorie $\mathrm{M}$, et al: The Romanian national program for liver transplantation -852 procedures in 815 patients over 17 years (2000-2017): A continuous evolution to success. Chirurgia (Bucur) 112: 229-243, 2017.

8. Chen RJ, Fang JF, Lin BC, Hsu YP, Kao JL and Chen MF: Factors determining operative mortality of grade $\mathrm{V}$ blunt hepatic trauma. J Trauma 49: 886-891, 2000.

9. Scheeren WL and Saugel B: Management of Intraoperative Hypotension: Prediction, prevention and personalization. In: Annual Update in Intensive Care and Emergency Medicine. Vincent JL (ed). Springer, p89-97, 2018.

10. Gupta B, Garg N and Ramachandran R: Vasopressors: Do they have any role in hemorrhagic shock? J Anaesthesiol Clin Pharmacol 33: 3-8, 2017.

11. Van der Linden P: Management of uncontrolled hemorrhagic shock: Toward a new clinical approach. Anesthesiology 107: 529-530, 2007.
12. Arabi Y, Venkatesh S, Haddad S, Al Shimemeri A and Al Malik S: A prospective study of prolonged stay in the intensive care unit: Predictors and impact on resource utilization. Int J Qual Health Care 14: 403-410, 2002.

13. Uchida K, Nishimura T, Hagawa N, Kaga S, Noda $T$, Shinyama N, Yamamoto H and Mizobata Y: The impact of early administration of vasopressor agents for the resuscitation of severe hemorrhagic shock following blunt trauma. BMC Emerg Med 20: 26, 2020

14. Rubenson Wahlin R, Nelson DW, Bellander BM, Svensson M, Helmy A and Thelin EP: Prehospital intubation and outcome in traumatic brain injury-assessing intervention efficacy in a modern trauma cohort. Front Neurol 9: 194, 2018.

15. Pepe PE, Roppolo LP and Fowler RL: Prehospital endotracheal intubation: Elemental or detrimental? Crit Care 19: 121, 2015.

16. Gravesteijn BY, Sewalt CA, Nieboer D, Menon DK, Maas A, Lecky F, Klimek M and Lingsma HF; CENTER-TBI collaborators: Tracheal intubation in traumatic brain injury: A multicentre prospective observational study. Br J Anaesth 125: 505-517, 2020.

17. Okabe Y: Risk factors for prolonged mechanical ventilation in patients with severe multiple injuries and blunt chest trauma: A single center retrospective case-control study. Acute Med Surg 5: 166-172, 2018.

18. McQuilten ZK, Wood EM, Bailey M, Cameron PA and Cooper DJ: Fibrinogen is an independent predictor of mortality in major trauma patients: A five-year statewide cohort study. Injury 48: 1074-1081, 2017.

19. Hayakawa M: Dynamics of fibrinogen in acute phases of trauma. J Intensive Care 5: 3, 2017.

20. Schlimp CJ, Ponschab M, Voelckel W, Treichl B, Maegele M and Schöchl H: Fibrinogen levels in trauma patients during the first seven days after fibrinogen concentrate therapy: A retrospective study. Scand J Trauma Resusc Emerg Med 24: 29, 2016.

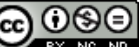

This work is licensed under a Creative Commons Attribution-NonCommercial-NoDerivatives 4.0 International (CC BY-NC-ND 4.0) License. 